\title{
pH matters: the influence of the catalyst ink on the oxygen reduction activity determined in thin film rotating disk electrode measurements
}

\author{
Masanori Inaba ${ }^{\mathrm{a}, \mathrm{b}}$, Jonathan Quinson ${ }^{\mathrm{a}}$, and Matthias Arenz ${ }^{\mathrm{a}, \mathrm{c}, *}$
}

${ }^{a}$ Nano-Science Center, Department of Chemistry, University of Copenhagen, Universitetsparken

5, DK-2100 Copenhagen Ø, Denmark

${ }^{\text {b}}$ Toyota Central R\&D Labs., Inc., Nagakute, Aichi 480-1192, Japan

'Department of Chemistry and Biochemistry, University of Bern, Freiestrasse 3, CH-3012 Bern, Switzerland

*Corresponding author

University of Bern, Freiestrasse 3, CH-3012 Bern, Switzerland

Phone: +41316315384

Email: matthias.arenz@dcb.unibe.ch 


\begin{abstract}
We investigated the influence of the ink properties of proton exchange membrane fuel cell (PEMFC) catalysts on the oxygen reduction reaction (ORR) activity determined in thin film rotating disk electrode (TF-RDE) measurements. It was found that the adaption of a previously reported ink recipe to home-made catalysts does not lead to satisfying results, although reported work could be reproduced using commercial catalyst samples. It is demonstrated that the $\mathrm{pH}$ of the catalyst ink, which has not been addressed in previous TF-RDE studies, is an important parameter that needs to be carefully controlled to determine the intrinsic ORR activity of high surface area catalysts.
\end{abstract}

\title{
Keywords
}

Oxygen reduction reaction

Platinum

Carbon supported catalyst

Rotating disc electrode

Catalyst ink

\section{Introduction}

Rotating disk electrode (RDE) measurements are a popular approach to test the performance of electrocatalysts in so-called electrochemical half-cell measurements [1-5]. Originally the method was developed for planar electrodes, where it enables defined mass transport conditions of a reactant that is dissolved in the electrolyte to the electrode surface. Using hydrodynamics, it is then possible to extract the "kinetics" from the measured current densities. One of the main assumptions for such a kinetic analysis, see ref. [6] for details, is that the mass transport to each 
point of the catalyst surface is identical.

With increasing interest in proton exchange membrane fuel cells (PEMFCs) there was a need for a fast reliable method to screen-test different catalysts, especially for the oxygen reduction reaction (ORR), the cathode process in PEMFCs. This need was addressed by applying a thin catalyst layer onto glassy carbon (GC) electrodes in a RDE configuration, the so-called thin-film (TF-) RDE method [1]. The TF-RDE method became extremely popular in PEMFC research and is now used as standard to compare different catalysts, and not least to substantiate claims of critical activity improvements.

However, as discussed in the work by Gasteiger et al. [2], the need for benchmarking catalysts became apparent with the comparison of activities reported by different groups: for similar catalysts, differences in ORR activities of one order of magnitude were reported. Such variations are indeed critical not only as they prove that comparisons to the literature are difficult; but because they also pose the question of the "real" intrinsic ORR activity of Pt catalysts.

Since then several groups worked on procedures to systematically analyze the ORR activity of fuel cell catalysts from TF-RDE measurements [3,7-13]. Especially, during the last few years, the influence of catalyst layer quality on measured ORR activity and its reproducibility has been highlighted. Several thin film fabrication approaches to obtain homogeneous catalyst layers were reported [11-13], showing that the optimization of the catalyst ink properties and the film drying method play a key role in the assessment of ORR activities of high surface area catalysts.

Most recently, researchers at the national renewable energy laboratory (NREL) characterized catalyst films fabricated by using several ink formulations and film-drying techniques [5]. According to their report, the highest quality films (based on homogeneity assessed by microscopy techniques as well as resulting ORR activity values) for a commercial TKK 46 
wt. \% Pt/C catalyst were obtained with high reproducibility by adding isopropanol (IPA) (25 \% in volume ratio) to a catalyst ink containing only pure water and the $\mathrm{Pt} / \mathrm{C}$ catalyst powder followed by drying the ink on GC electrodes in an IPA atmosphere (Nafion-free stationary IPA drying (NF-SIPAD) technique). The measured ORR specific activity (SA) from these catalyst films was significantly higher than the values reported in previous reports [2,8,12,14-17].

In the work presented here, we tried to adapt the reported ink recipe and film-drying method to home-made catalyst that were synthesized via a colloidal approach that aims at enabling systematic studies of high surface area catalysts [18]. Our results demonstrate that a direct transfer leads to inferior results as compared to using pure water to prepare the catalyst inks although the reported findings could be reproduced. It is argued that, similar to the preparation of membrane electrode assemblies (MEAs) [19], the $\mathrm{pH}$ of the catalyst ink is an important parameter that needs to be carefully controlled when evaluating ORR activity of high surface area catalysts via the TF-RDE technique.

\section{Experimental}

\subsection{Chemicals and gases}

The following chemicals were used in the catalyst synthesis and characterization: ethylene glycol (EG, 99.8 \%, Sigma-Aldrich), sodium hydroxide (NaOH, 98.9 \%, Fisher Chemical), hexachloroplatinic(IV) acid hexahydrate $\left(\mathrm{H}_{2} \mathrm{PtCl}_{6} \cdot 6 \mathrm{H}_{2} \mathrm{O}, 99.9 \%\right.$, Alfa Aesar), $30 \%$ hydrochloric acid ( $\mathrm{HCl}$, Suprapur, Merck), 60 \% nitric acid $\left(\mathrm{HNO}_{3}\right.$, Suprapur, Merck) and acetone (99.5+ \%, Alfa Aesar). Two kinds of commercial carbon black, Vulcan XC72R (Cabot Corporation, BET area: $235 \mathrm{~m}^{2} \mathrm{~g}^{-1}$ ) and Ketjen EC-300J (AkzoNobel Polymer Chemistry, BET area: $795 \mathrm{~m}^{2} \mathrm{~g}^{-1}$ ), were employed as carbon support in the catalyst synthesis. Deionized (DI) water (resistivity $>18.2 \mathrm{M} \Omega \cdot \mathrm{cm}$, total organic carbon (TOC) $<5 \mathrm{ppb}$ ) from a Milli-Q system 
(Millipore) was used for acid/base dilutions, catalyst ink formulation, and the electrochemical cell cleaning. Isopropanol (IPA, 99.7+ \%, Alfa Aesar), $70 \%$ perchloric acid $\left(\mathrm{HClO}_{4}\right.$, Suprapur, Merck), and potassium hydroxide hydrate $\left(\mathrm{KOH} \cdot \mathrm{H}_{2} \mathrm{O}\right.$, Suprapur, Merck) were used for catalyst ink formulation and electrolyte preparation. The following gases from Air Liquide were used in electrochemical measurements: $\mathrm{Ar}(99.999 \%), \mathrm{O}_{2}$ (99.999 \%), $\mathrm{CO}(99.97 \%)$, and $\left(\mathrm{H}_{2}\right.$ $99.999 \%)$.

\subsection{Synthesis of $50 \mathrm{wt} . \% \mathrm{Pt} / \mathrm{C}$ catalysts}

50 wt. \% Pt/C electrocatalysts were synthesized via the tool-box approach we previously reported [18]. The synthesis method of the electrocatalyst consists of two main steps: a suspension of colloidal Pt nanoparticles (NPs) is prepared via an alkaline ethylene glycol (EG) route [20], and then the NPs are deposited onto carbon support.

A colloidal suspension of Pt NPs ca. $2 \mathrm{~nm}$ was synthesized by mixing $4 \mathrm{~mL}$ of a solution of $\mathrm{NaOH}$ at $0.4 \mathrm{M}$ in EG with $4 \mathrm{~mL}$ of a solution of $\mathrm{H}_{2} \mathrm{PtCl}_{6} \cdot 6 \mathrm{H}_{2} \mathrm{O}$ at $40 \mathrm{mM}$ in EG in a microwave reaction vessel. The mixture was heated for 3 minutes at $160{ }^{\circ} \mathrm{C}$ with a microwave reactor (CEM Discover SP, $100 \mathrm{~W}$ heating power). The solution initially yellow turned dark during the heating.

In order to support the Pt NPs onto a carbon support, $30 \mathrm{~mL}$ of $1 \mathrm{M} \mathrm{HCl}$ solution was added to $7.3 \mathrm{~mL}$ of the colloidal Pt NPs suspension for precipitation. The mixture was centrifuged at 2400 relative centrifugal force (4000 rotations per minute (rpm), Sigma 2-5) for 5 minutes and the supernatant solvent discarded. This washing/centrifugation with $1 \mathrm{M} \mathrm{HCl}$ was repeated twice. Then the Pt NPs were re-dispersed in $7 \mathrm{~mL}$ of acetone, and $27.5 \mathrm{mg}$ of carbon black (Vulcan XC72R or Ketjen EC-300J) was added to the suspension. By sonicating the mixture in an ultrasonic bath (Ultrasonic cleaner, VWR) for 1 hour, the acetone was evaporated and a dried powder of Pt NPs supported on carbon (Pt/C) was obtained. The dried powder was re-dispersed 
into water and sonicated for 10 minutes. The Pt/C catalyst powder was filtered and washed with $200 \mathrm{~mL}$ of DI water, and then dried at $100{ }^{\circ} \mathrm{C}$ in air.

\subsection{Characterization of homemade Pt/C catalysts}

The particle size distribution of the Pt NPs obtained from the colloidal synthesis was determined by small-angle X-ray scattering (SAXS) measurements as previously reported [21,22] with a SAXSLab instrument installed at the Niels Bohr Institute of the University of Copenhagen. The particle size distribution of Pt NPs immobilized on the carbon support was determined by transmission electron microscopy (TEM, JEM-2100, JEOL, $200 \mathrm{kV}$ ). The size analysis was performed by measuring the size of typically 300 NPs by taking images at 3 different magnifications in 5 different areas of the TEM grid. The Pt loading of the catalyst was evaluated by inductively coupled plasma mass spectrometry (ICP-MS) as previously reported [21]. Measurements were performed at the Max Planck Institute for Iron Research (MPIE) in Dusseldorf, Germany. For sample preparation $100 \mu \mathrm{L}$ of the catalyst ink was digested in $4 \mathrm{~mL}$ of aqua regia (mixture of $30 \% \mathrm{HCl}$ and $65 \% \mathrm{HNO}_{3}$ in a volume ratio of $1: 3$ ) for 90 minutes on a hot plate at ca. $100{ }^{\circ} \mathrm{C}$. The final volume was finally adjusted to $10 \mathrm{~mL}$ with DI water. The sample solutions were filtered to remove residual carbon before the measurements. As the synthesis is very reproducible, we refer for the results of this basic characterization to our previous work [21,22], from which it can be seen that the physical properties of our home-made catalyst are indeed very similar to the commercial TKK catalyst used for comparison, see below (size distribution determined by TEM are $1.9 \pm 0.6 \mathrm{~nm}$ vs $2.2 \pm 0.6 \mathrm{~nm}$ for home-made and TKK catalyst, respectively).

\subsection{Ink formulation for homemade Pt/C catalysts}

$4.4 \mathrm{mg}$ of homemade $50 \mathrm{wt}$ \% Pt/Vulcan or Pt/Ketjen catalyst powder was mixed with $8 \mathrm{~mL}$ of IPA:DI water (1:3, v:v) mixed solvent. A small amount of $1 \mathrm{M} \mathrm{KOH}$ solution (8 40 $\mu \mathrm{L})$ was 
added as needed to adjust the $\mathrm{pH}$ of the ink. The glass vial containing the mixture was placed in an ultrasonic bath filled with cold water $\left(<5^{\circ} \mathrm{C}\right)$ and sonicated for 15 minutes.

\subsection{Ink formulation for commercial Pt/C catalyst}

$5.97 \mathrm{mg}$ of commercial $46.5 \mathrm{wt}$ \% Pt/C catalyst (TEC10E50E, TKK) powder was mixed with $10 \mathrm{~mL}$ of IPA:DI water (1:3, v:v) mixed solvent. A few $\mu \mathrm{L}$ of $1 \mathrm{M} \mathrm{KOH}$ solution or $0.1 \mathrm{M}$ $\mathrm{HClO}_{4}$ solution was added as needed to adjust $\mathrm{pH}$ of the ink. The glass vial containing the mixture was placed in the ultrasonic bath filled with cold water $\left(<5^{\circ} \mathrm{C}\right)$ and sonicated for 15 minutes. Subsequently the mixture was dispersed using a horn sonicator (Q500, QSonica) for 1 minute.

\subsection{Characterization of catalyst inks}

The $\mathrm{pH}$ of the catalyst ink was measured using a pH meter (Symphony SP70P, VWR) with a glass electrode (InLab Routine, Mettler Toledo). The aggregate size in the catalyst ink and the zeta potential of the catalyst particles were measured by dynamic light scattering (DLS) and electrophoretic light scattering (ELS), respectively (Litesizer ${ }^{\mathrm{TM}}$ 500, Anton Paar). For the DLS measurements, $30 \mu \mathrm{L}$ of catalyst inks were diluted by $1 \mathrm{~mL}$ of DI water 1 hour after the ink preparation. For the ELS measurements, $20 \mu \mathrm{L}$ of the catalyst inks were diluted by $1 \mathrm{~mL}$ of $\mathrm{pH}$ adjusted electrolyte solutions (mixture of $\mathrm{KCl}, \mathrm{HCl}$, and $\mathrm{KOH}$, total ion concentration of $40 \mathrm{mM}$, $\mathrm{pH}=2.9,5.1,7.2,8.7,10.8)$

\subsection{Catalyst thin film fabrication}

The catalyst thin film was fabricated based on the "Nafion-free stationary IPA drying (NF-SIPAD) technique” reported by Shinozaki et al. [5]. A glassy carbon (GC) disc electrode (5 $\mathrm{mm}$ in diameter, $0.196 \mathrm{~cm}^{2}$ in geometric area) was polished to mirror finish using alumina oxide paste (0.3 and $0.05 \mu \mathrm{m}$ AP-D suspension, Struers), and cleaned ultrasonically in DI water. A 10 $\mu \mathrm{L}$ aliquot of the catalyst ink was pipetted onto the GC electrode leading to a Pt loading of 14 
$\mu \mathrm{g}_{\mathrm{Pt}} \mathrm{cm}^{-2}$. Subsequently the GC electrode was placed in a beaker $(250 \mathrm{~mL})$ with a small glass vial filled with IPA. The beaker with the electrode and IPA was covered by a glass dish, and kept at room temperature to gradually ( $\sim 2$ hours) dry the ink under IPA atmosphere. To assess the film quality, after the electrochemical measurements, the catalyst films on the GC electrodes were transferred on a tissue paper by pressing the electrodes on the wetted paper and photographs of the transferred catalyst films were taken.

\subsection{Electrochemical cell setup}

An in-house Teflon cell based on a three-compartment configuration was employed in all electrochemical measurements [23]. A platinum mesh was used as a counter electrode. A saturated calomel electrode (B3510+, Schott) was employed as reference electrode and placed in a sub-compartment separated by a Nafion membrane in order to avoid the diffusion of chloride ions into the main compartment. All potentials in this study are referred to the reversible hydrogen electrode (RHE) potential, which was experimentally determined for each measurement series. The Teflon cell and components were soaked in mixed acid $\left(\mathrm{H}_{2} \mathrm{SO}_{4}: \mathrm{HNO}_{3}\right.$. $=1: 1$, v:v) overnight. Subsequently the Teflon cell and other components were rinsed thoroughly by DI water, and boiled in DI water twice. Between electrochemical experiments, the Teflon cell and components were boiled and kept in DI water.

\subsection{Electrochemical measurements}

All electrochemical measurements were performed using a computer controlled, potentiostat (ECi 200, NordicElectrochemistry) and the Teflon cell with an RDE rotator (EDI101, Radiometer Analytical). The measurements were performed in $0.1 \mathrm{M} \mathrm{HClO}_{4}$ solution at $20{ }^{\circ} \mathrm{C}$. Prior to the measurements, the electrolyte was de-aerated by purging with Ar gas, and the catalysts were cleaned by potential cycles between 0.05 and $1.20 \mathrm{~V}$ at a scan rate of $500 \mathrm{mV} \mathrm{s}^{-1}$ until a stable cyclic voltammogram could be observed. The electrochemical surface area 
(ECSA) of the catalyst was determined by conducting CO stripping voltammetry [3]. The working electrode was held at $0.05 \mathrm{~V}$ during a $\mathrm{CO}$ purge through the electrolyte followed by an Ar purge. The ECSA was estimated from the charge recorded at a scan rate of $50 \mathrm{mV} \mathrm{s}^{-1}$ using conversion coefficient of $396 \mu \mathrm{C} \mathrm{cm}{ }^{-2}$ pt. In order to determine the ORR activity, linear sweep voltammetry (LSV, positive scan) was conducted in $\mathrm{O}_{2}$ saturated electrolyte at a scan rate of 50 $\mathrm{mV} \mathrm{s}^{-1}$ and a rotation speed of $1600 \mathrm{rpm}$. The polarization curves were corrected for the nonfaradaic background by subtracting the cyclic voltammograms (CVs) recorded in Ar-purged electrolyte at the identical scan rate. Furthermore, the solution resistance $\left(R_{\text {sol }}\right)$ between the working electrode and the Luggin capillary ( $23 \Omega$ ) was determined using an AC signal (5 kHz, $5 \mathrm{mV}$ ) and thereafter compensated for using the potentiostat's analog positive feedback scheme. The resulting effective solution resistance was less than $3 \Omega$ for each experiment. The ORR kinetic current density $i_{k}$ was determined using Koutecky-Levich equation described as:

$$
\frac{1}{i}=\frac{1}{i_{k}}+\frac{1}{i_{d}}
$$

where $i$ and $i_{d}$ are measured current density and diffusion limiting current density, respectively. In this study, the ORR activity of the catalysts is always evaluated at $0.9 \mathrm{~V}$ vs. RHE.

\section{Results and discussion}

3.1. Impact of ink formulation and catalyst film fabrication in RDE measurement of commercial catalyst

As mentioned in the introduction, the aim of this study was to transfer the ink recipe (IPA: $\mathrm{H}_{2} \mathrm{O}(1: 3, \mathrm{v}: \mathrm{v})$ as solvent) reported in a recent study by NREL to homemade catalysts. We started our investigations with reproducing reported findings. For this we used the same commercial catalyst as used by NREL and applied the NF-SIPAD technique for preparing catalyst films. As reported in the literature, a nicely dispersed catalyst ink and uniform catalyst 
films without agglomeration were obtained (see below). When the ECSA and ORR activity values of these catalyst films are compared with those from our own previous work with the same catalyst, but where we prepared the catalyst film by dispersing the catalyst in pure water followed by drying the ink on GC electrodes under $\mathrm{N}_{2}$ gas flow [17], the NREL's findings are confirmed, see Fig. 1. A significant increase in ORR specific activity is achieved by applying NF-SIPAD method as compared to drying the catalyst ink under $\mathrm{N}_{2}$ gas flow. It should be noted here that we kept RDE measurement conditions and experimental procedures employed identical to our previous experiments [17].

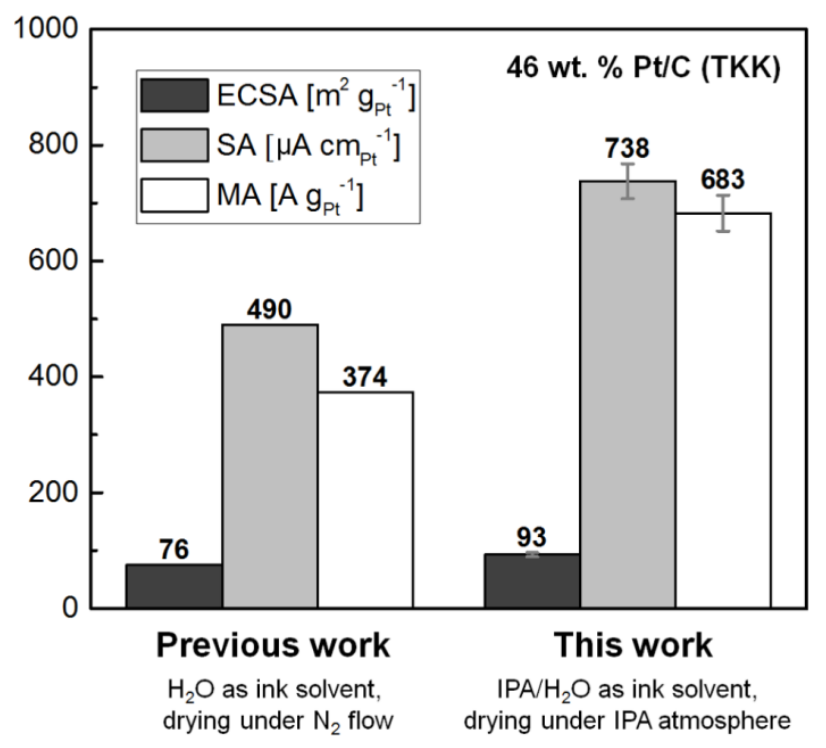

Fig. 1. Comparison of our previous data and the presented work where NF-SIPAD technique was employed for the catalyst film fabrication. In both work, the commercial $46 \mathrm{wt}$ \% Pt/C catalyst (TKK) was measured by RDE employing identical condition and experimental procedure. Our previous data is taken from reference [17].

Interestingly, not only the ORR specific activity depends on the ink formulation and drying procedure, but also a significant increase in ECSA was observed by adding IPA to the catalyst inks. The same result was reported by Takahashi et al. [8]. They varied the ratio between water 
and IPA to determine the optimal IPA content. Thus they could increase the determined ECSA by $\sim 20 \%$ as compared to IPA-free inks. However, the mechanism for the increase in ECSA has not been clarified. A possible explanation for this phenomenon is that upon addition of IPA the wettability of the carbon support increases enabling the electrolyte solution to access $\mathrm{Pt}$ nanoparticles located in micro-pores of the carbon support, which otherwise would not participate in reactions.

\subsection{Application of NF-SIPAD method to Pt/C catalyst synthesized by the tool-box approach}

To conduct systematic studies of high surface area catalysts, we recently introduced a catalyst synthesis platform which we dubbed “tool-box approach”[18]. In this approach carbon supported, Pt based catalysts are prepared from a colloidal suspension of well-defined Pt NPs synthesized by an alkaline EG method. A scheme of the synthesis platform is shown in Fig. 2a. Monodisperse Pt NPs with a diameter around $2 \mathrm{~nm}$ are synthesized in alkaline EG [18]. The Pt NPs are washed by $1 \mathrm{M} \mathrm{HCl}$ solution, re-dispersed in acetone, and then supported on a carbon material. The Pt particles are free of any strongly binding surfactants or other protection agents [24], which make them ideal for electrochemical applications due to their clean surface. No heat treatment steps are required during the synthesis. Using this approach, parameters such as Pt to carbon ratio (inter-particle distance between the NPs), and support material can be independently varied and their effect on the catalytic properties independently studied $[18,22]$. As examples of the catalysts obtained from this synthesis approach, TEM images of $50 \mathrm{wt}$ \% Pt/Vulcan and 50 wt. \% Pt/Ketjen are shown in Fig. 2b and Fig. 2c, respectively. 
(a)

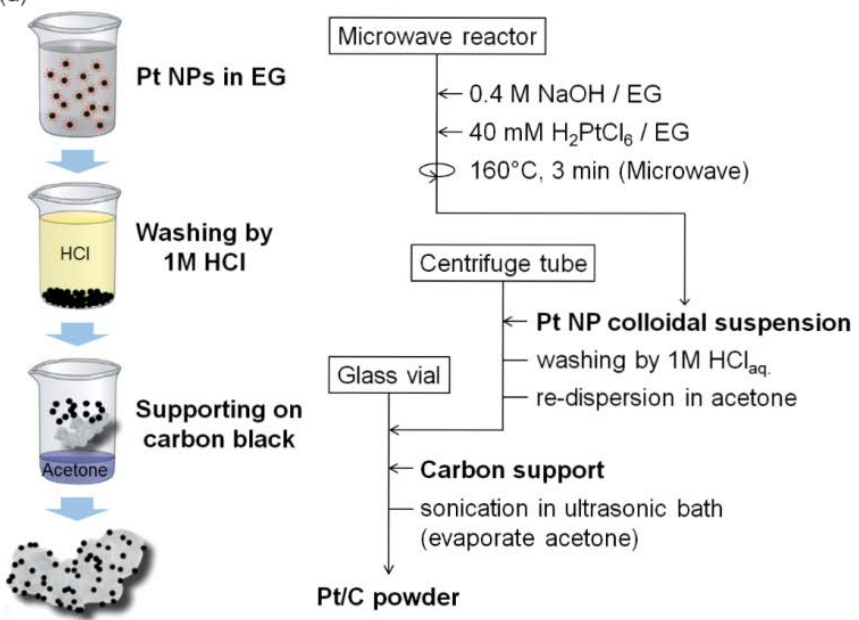

(b)

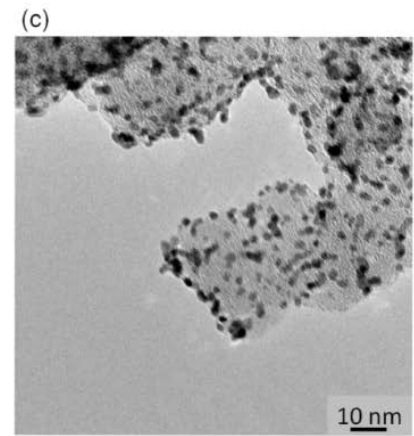

Fig. 2. (a) Scheme of the Pt/C catalyst synthesis via tool-box synthesis approach and TEM images of (b) 50 wt. \% Pt/Vulcan catalys and (c) 50 wt. \% Pt/Ketjen catalyst that are obtained from the synthesis approach.

To study the ink properties of such synthesized catalysts and its influence on the electrocatalytic performances, the 50 wt. \% Pt/Vulcan catalyst was prepared by our tool-box approach and thin catalyst films were fabricated from a catalyst ink by applying the NF-SIPAD method, which was effective for fabricating uniform catalyst films from the commercial TKK 46 wt. \% Pt/C catalyst. However, the dispersion of the catalyst ink from the 50 wt. \% Pt/Vulcan catalyst was poor and the catalyst easily agglomerated (see Fig. 3). As a consequence, large agglomerations were seen in the prepared catalyst thin films. Interestingly, in contrast to the commercial catalyst the quality of the films prepared from IPA containing ink was significantly 
lower than films prepared from IPA-free ink.

46 wt. \% Pt/C (TKK) IPA/ $\mathrm{H}_{2} \mathrm{O}$ ink IPA dry

(a)

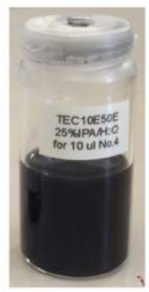

(b)

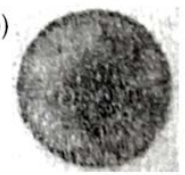

50 wt. \% Pt/Vulcan

$\mathrm{H}_{2} \mathrm{O}$ ink $\mathrm{N}_{2}$ flow dry

(c)

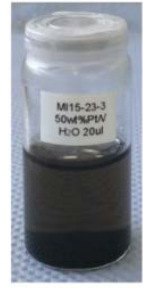

(d)

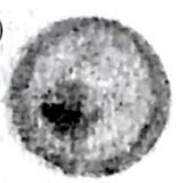

50 wt. \% Pt/Vulcan

IPA/ $\mathrm{H}_{2} \mathrm{O}$ ink IPA dry

(e)

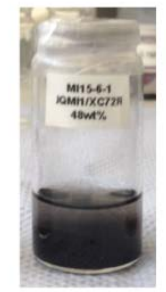

(f)

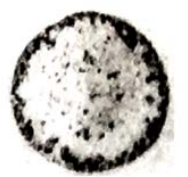

Fig. 3. Photographs of catalyst inks and catalyst films fabricated from each catalyst ink: (a)(b) the commercial 46 wt. \% Pt/C catalyst (TKK), IPA:DI water (1:3, v:v) as ink solvent, ink drying in IPA atmosphere, (c)(d) the homemade 50 wt. \% Pt/Vulcan catalyst, $\mathrm{H}_{2} \mathrm{O}$ as ink solvent, ink drying under $\mathrm{N}_{2}$ flow, (e)(f) the homemade50 wt. \% Pt/Vulcan catalyst, IPA:DI water (1:3, v:v) as ink solvent, ink drying in IPA atmosphere. The catalyst films were fabricated on the GC electrodes and thereafter transferred on a paper before taking the photographs.

In order to investigate the difference between the observed behavior of the commercial 46 wt. \% Pt/C catalyst and our homemade 50 wt. \% Pt/Vulcan catalyst, the $\mathrm{pH}$ values of the catalyst inks of both catalysts were compared. It was seen that the ink of the $50 \mathrm{wt}$ \% Pt/Vulcan catalyst was highly acidic $(\mathrm{pH}=3.2)$, whereas the ink of the commercial $46 \mathrm{wt} . \% \mathrm{Pt} / \mathrm{C}$ catalyst was slightly alkaline $(\mathrm{pH}=8.5)$. As the carbon support itself is not acidic, we relate this finding to residual $\mathrm{HCl}$, which was used to wash the Pt NPs in the catalyst synthesis (the influence of $\mathrm{HCl}$ on the ink properties of the commercial $46 \mathrm{wt}$ \% Pt/C catalyst is described in the supporting information). We hypothesize that it is the $\mathrm{pH}$ of a catalyst ink that controls the zeta potential of the catalyst particles (carbon particles with the active Pt centers) and thereby 
dispersion of the ink.

\subsection{Influence of $p H$ of catalyst inks on the ink formulation and the catalyst film fabrication}

To test this hypothesis, the $\mathrm{pH}$ of catalyst inks prepared from the homemade $50 \mathrm{wt}$ \% Pt/Vulcan catalyst was systematically varied by adding small amounts of $1 \mathrm{M} \mathrm{KOH}$ aqueous solution to the ink. Without adding $\mathrm{KOH}$, the catalyst ink is acidic $(\mathrm{pH}=4.3)$ and color of the ink is pale black (Fig. 4a). The stability of the ink is low, thus precipitates are seen in the ink 1 hour after sonication. The agglomeration of the catalyst progressed during the film drying, and large aggregates are seen in the catalyst film obtained from the acidic ink (Fig. 4b). When the $\mathrm{pH}$ of the ink is increased to approximately neutral values $(\mathrm{pH}=8.0)$ by adding the $\mathrm{KOH}$ solution, the catalyst ink becomes more stable and slightly more blackish than the acidic ink (Fig. 4c). The agglomeration of the catalyst during the film drying is inhibited and uniform catalyst film can be obtained (Fig. 4d). When the $\mathrm{pH}$ of the ink is further increased to alkaline values $(\mathrm{pH}=11.3)$, the catalyst ink becomes considerably more blackish than the neutral or acidic ink (Fig. 4e). This catalyst ink is so stable that no precipitates are observed even 1 day after the ink preparation. From the alkaline catalyst ink, highly uniform catalyst films can be fabricated with high reproducibility (Fig. 4f).

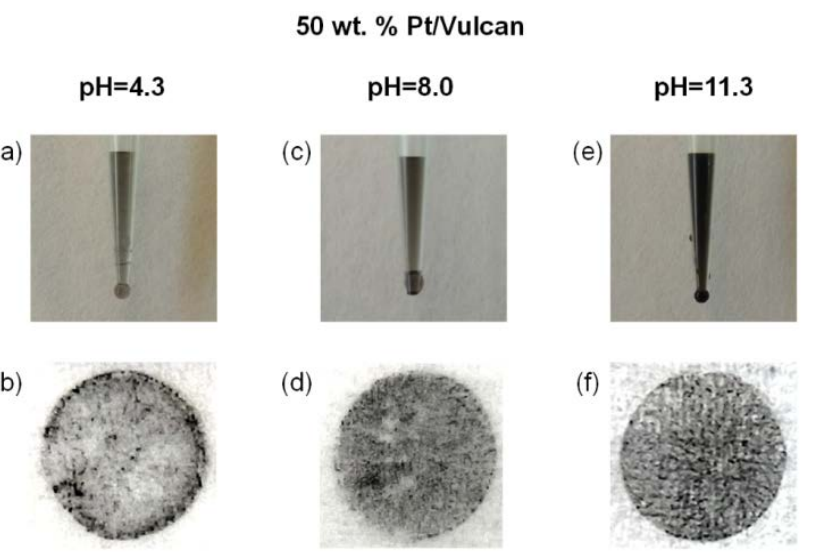

Fig. 4. Photographs of catalyst inks of the homemade $50 \mathrm{wt}$. \% Pt/Vulcan catalyst with different pH and catalyst films fabricated from each catalyst ink: (a)(b) ink $\mathrm{pH}=4.3$, without adding $\mathrm{KOH}$, (c)(d) ink $\mathrm{pH}=8.0, \mathrm{KOH}$ added, (e)(f) ink $\mathrm{pH}=11.3, \mathrm{KOH}$ added. The catalyst films were fabricated on the GC electrodes and thereafter transferred on a paper before taking the photographs. 
The catalyst films fabricated from the three catalyst inks with different $\mathrm{pH}(\mathrm{pH}=4.3,8.0$, 11.3) were also characterized by RDE measurements. It should be emphasized that the chloride concentration in all three inks is the same. In the cyclic voltammograms measured in Ar-purged electrolyte, no significant difference is observed. The cyclic voltammograms obtained from the catalyst films prepared from the acidic ink $(\mathrm{pH}=4.3)$ and alkaline ink $(\mathrm{pH}=11.3)$ are shown in Fig. 5a. In the hydrogen under potential deposition (H-UPD) potential region $(0.05-0.30 \mathrm{~V}$ vs. RHE), both hydrogen adsorption peaks and hydrogen desorption peaks are almost identical. In the platinum redox region (0.5 - $1.1 \mathrm{~V}$ vs. RHE), platinum oxidation and reduction peaks are also quite similar, although in the voltammograms of the acidic ink the platinum oxidation peak is slightly inclined and the current density at $1.1 \mathrm{~V}$ vs RHE is higher than that of the alkaline ink. This finding is probably related to trace amounts of impurities on the platinum surface, which are not completely removed by the potential cycling conducted before recording the shown voltammograms to clean the Pt surface. It is also observed that during the potential cycling of the more uniform catalyst films, the "steady state" shape of the voltammogram is reached faster than for the less uniform catalyst films. 

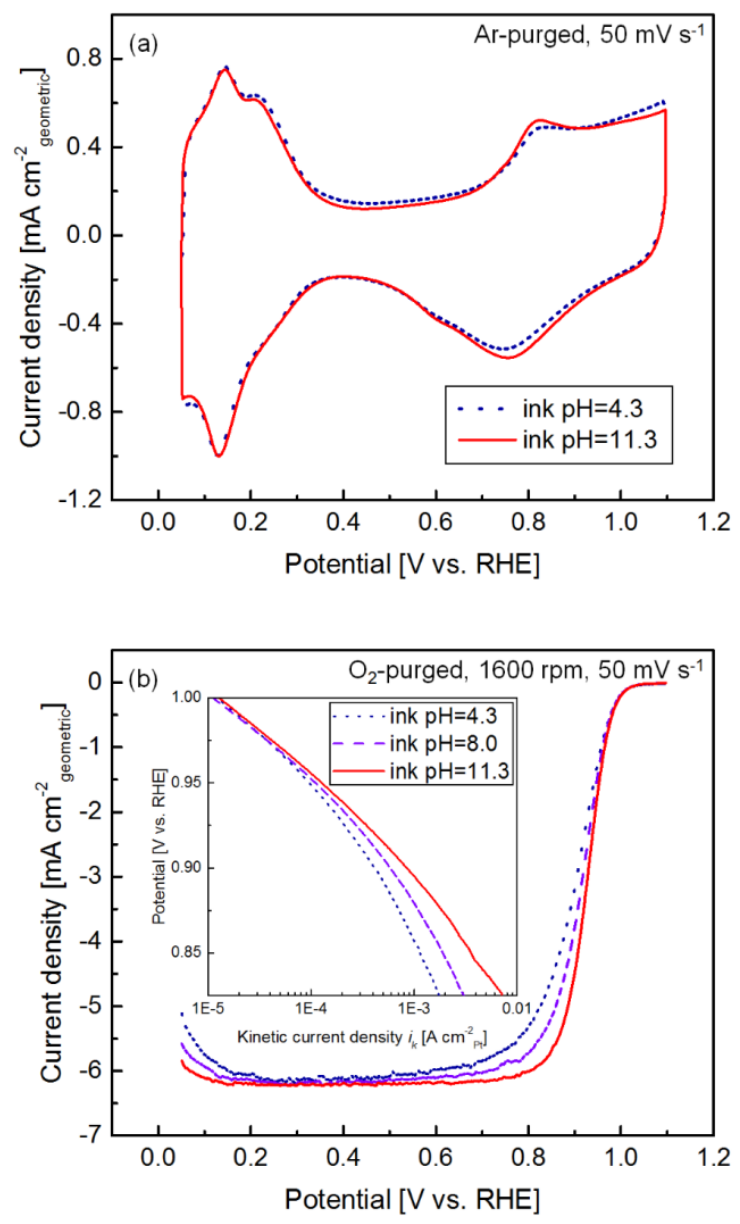

Fig. 5. Comparison of voltammograms for the catalyst films fabricated from the catalyst inks of the homemade 50 wt. \% Pt/Vulcan catalyst with different $\mathrm{pH}(\mathrm{pH}=4.3,8.0,11.3)$ : (a) cyclic voltammograms in Ar-purged $0.1 \mathrm{M} \mathrm{HClO}_{4}$ at the scan rate of $50 \mathrm{mV} \mathrm{s}^{-1}$. The neutral ink $(\mathrm{pH}=$ 8.0) is not shown for the clarity. (b) linear sweep voltammograms (positive scans) in $\mathrm{O}_{2}$-purged 0.1 $\mathrm{M} \mathrm{HClO}_{4}$ at the scan rate of $50 \mathrm{mV} \mathrm{s}{ }^{-1}$ and the rotation speed of $1600 \mathrm{rpm}$. $\mathrm{R}_{\text {sol }}$ and background were corrected. Inset is Tafel plot obtained from the linear sweep voltammograms.

Unlike the cyclic voltammetry in Ar-purged electrolyte, significant differences are observed in the ORR measurements of the various thin films prepared by catalyst inks with different $\mathrm{pH}$. Linear sweep voltammograms from the catalyst films of the three catalyst inks recorded in $\mathrm{O}_{2}$-purged electrolyte are shown in Fig. 5b. The current density increases with increasing $\mathrm{pH}$ of 
the catalyst ink, especially in the mixed kinetic-diffusion controlled region $(0.75-1.0 \mathrm{~V}$ vs. RHE) used to determine the ORR activity. In addition, the elbow of the voltammogram around $0.75-0.85 \mathrm{~V}$ vs. RHE becomes sharper with increasing ink $\mathrm{pH}$. The limiting current plateau is reached near $0.6 \mathrm{~V}$ vs. RHE when the ink $\mathrm{pH}$ is 4.3 , whereas it is reached near $0.75 \mathrm{~V}$ vs. RHE when the ink $\mathrm{pH}$ is 11.3. Tafel plots obtained from the positive going linear sweep voltammograms are depicted in the inset of Fig. 5b. It is seen that the kinetic current densities of the three catalyst films are almost identical at high potentials ( $>0.95 \mathrm{~V}$ vs. RHE). At lower potentials, however, the kinetic current density increases with increasing catalyst ink $\mathrm{pH}$, and the difference between the measured kinetic current densities of the three catalyst films increases with decreasing the potential. We assume that the reduced kinetic current densities obtained by using the acidic ink and the neutral ink are due to mass transport resistance, such as $\mathrm{O}_{2}$ diffusion resistance and protonic resistance, within the catalyst thin film. In previous qualitative analyses, the $\mathrm{O}_{2}$ diffusion resistance has been referred as a cause of the activity loss for the non-uniform catalyst films with coffee rings [5]. In our result, the uniformity of the catalyst thin film improves and the kinetic current density at $0.85 \mathrm{~V}$ vs. RHE significantly increases from $1.2 \mathrm{~mA} \mathrm{~cm}^{-2} \mathrm{Pt}$ to $1.9 \mathrm{~mA} \mathrm{~cm}^{-2} \mathrm{Pt}$ by increasing the catalyst ink $\mathrm{pH}$ from 4.3 to 8.0. Interestingly, the kinetic current density further increases to $3.6 \mathrm{~mA} \mathrm{~cm}{ }^{-2} \mathrm{pt}$ by increasing the catalyst ink pH to 11.3, even though there was no significant optical difference in the uniformity between the catalyst films fabricated from the neutral and the alkaline ink (Figs. 4df).

The addition of $\mathrm{KOH}$ to the catalyst ink changes its $\mathrm{pH}$, but leaves the chloride content constant. Our results therefore demonstrate that the $\mathrm{pH}$ of the catalyst ink can be a key parameter that needs to be optimized to fabricate uniform catalyst films and evaluate the ORR activity of high surface area catalysts while minimizing the influence of the mass transport resistance. The chloride ion concentration in the catalyst ink, although chloride is a 
well-established catalyst poison, seems not to have a direct influence on the ink stability and the uniformity of the prepared catalyst films. It should also be noted that extensive catalyst washing to remove residual $\mathrm{HCl}$ and to obtain $\mathrm{pH}$ neutral catalyst inks was found not to be sufficient to obtain optimal ink and film quality, since the best result was obtained by using the highly alkaline $(\mathrm{pH}=11.3)$ catalyst ink.

\subsection{Dependence of ECSA and ORR specific activity on $\mathrm{pH}$ of the catalyst inks}

To investigate the influence of the $\mathrm{pH}$ of the catalyst ink in more depth, various catalyst inks with different $\mathrm{pH}$ were prepared using the homemade 50 wt. \% Pt/Vulcan catalyst and the catalyst films fabricated from those inks were characterized by the RDE measurements. Three or four measurements were conducted for each catalyst ink, and ECSA and ORR specific activity at $0.9 \mathrm{~V}$ vs. RHE were analyzed for each measurement. The average values for each catalyst ink are plotted in Fig. 6ab. A clear trend is seen between the catalyst ink $\mathrm{pH}$ and the measured ORR specific activity. The specific activity increases with increasing ink $\mathrm{pH}$ and reaches a maximum at $\mathrm{pH}=11$ with an increase in ORR specific activity by roughly a factor of 5. When the catalyst ink $\mathrm{pH}$ is above 12 , the catalyst ink becomes unstable again and non-uniform catalyst films are obtained. Conversely, the ECSA exhibits no clear dependence of the catalyst ink $\mathrm{pH}$ as shown in Fig. 6b, although there is a certain spread between values obtained from the different catalyst inks. 

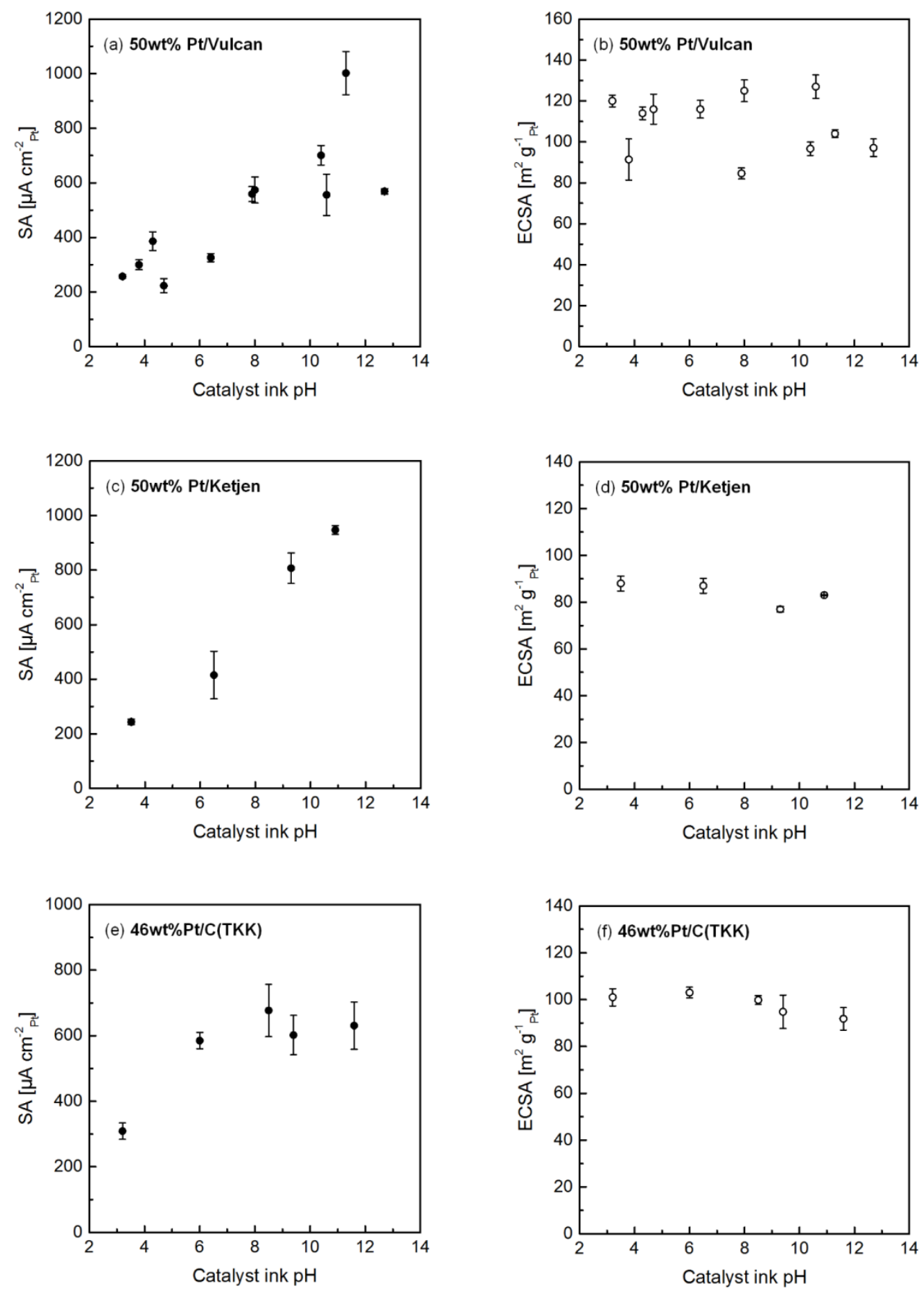

Fig. 6. Influence of pH of catalyst inks on SA at $0.9 \mathrm{~V}$ vs. RHE and ECSA: (a)(b) the homemade 50 wt. \% Pt/Vulcan catalyst, (c)(d) the homemade 50 wt. \% Pt/Ketjen catalyst, (e)(f) the commercial 46 wt. \% Pt/C catalyst (TKK). At least three measurements were conducted for each catalyst ink. 
The dependence of the catalyst ink $\mathrm{pH}$ is also confirmed in investigations using the homemade 50 wt. \% Pt/Ketjen catalyst, which was prepared by the same tool-box synthesis approach, but using a different carbon support. Without adding $\mathrm{KOH}$, the catalyst ink from this catalyst is highly acidic $(\mathrm{pH}=3.5)$ as well. The dispersion of the ink is unstable, and the catalyst agglomerates during the drying of the ink. Adding $\mathrm{KOH}$ solution, the catalyst ink becomes more stable and the uniformity of the prepared catalyst films is improved, although the change in the color of the ink is not as significant as in the case of the $50 \mathrm{wt}$ \% Pt/Vulcan catalyst ink. Average specific activity and ECSA values for each catalyst ink are plotted in Fig. 6cd. As in the case of the Pt/Vulcan catalyst, the specific activity increases with increasing ink $\mathrm{pH}$, while the ECSA remains constant. In agreement with previous observations [22], even though the same Pt NPs colloidal mother solution was used for synthesizing both the Pt/Vulcan and the Pt/Ketjen catalyst, the ECSA of the Pt/Ketjen catalyst is around $20 \%$ lower than that of the Pt/Vulcan catalyst. This effect is likely due to some Pt NPs being located in the micropores of the Ketjen black support rendering them inaccessible to the electrolyte solution [25].

The catalyst ink of the commercial TKK 46 wt. \% Pt/C catalyst was examined as well. In this case, without adding $\mathrm{KOH}$ the catalyst ink is slightly alkaline $(\mathrm{pH}=8.5)$, as previously mentioned. Therefore, to decrease the $\mathrm{pH}$ small amounts of $0.1 \mathrm{M} \mathrm{HClO}_{4}$ aqueous solution were added to the catalyst ink. It is seen that with decreasing the $\mathrm{pH}$, the catalyst ink becomes less stable and the resulting catalyst films less uniform; the ORR specific activity decreases, see Fig. 6e. On the other hand, increasing the ink $\mathrm{pH}$ by adding the $\mathrm{KOH}$ solution does not lead to improvements in the specific ORR activity. The ECSA is independent of the catalyst ink pH, as shown in Fig. 6f.

\subsection{Characterization of the catalyst inks by light scattering measurements}

To shed further light into the $\mathrm{pH}$ dependent ink properties and obtain quantitative physical 
data, we determined the average aggregate size of the catalyst particles in the catalyst inks using dynamic light scattering. Three catalyst inks with different $\mathrm{pH}$ (acidic, neutral, and alkaline) for the 50 wt. \% Pt/Vulcan catalyst and the 50 wt. \% Pt/Ketjen catalyst were measured, respectively. The results are shown in Fig. 7a. For both catalysts, the aggregate size decreases with increasing catalyst ink pH. This result can be related to the observed difference in the color of the catalyst inks seen in Fig. 4. It demonstrates that smaller agglomerates and a better dispersion with improved stability are reflected by a darker color, which thus can be used as a first control for ink stability.

In addition, the zeta potential of the catalyst particles was measured using electrophoretic light scattering. Zeta potential is an important indicative of ionically stabilized colloid systems. A large magnitude (usually $\pm 25 \mathrm{mV}$ ) of the zeta potential indicates a stable colloid system against coagulation [26]. As shown in Fig. 7b, for both catalysts, the catalyst particles have isoelectric point at $\mathrm{pH}=3 \sim 5$ and exhibit a negative zeta potential when $\mathrm{pH}$ is higher than the isoelectric point; the absolute value of the zeta potential increases with increasing $\mathrm{pH}$ and exceeds $25 \mathrm{mV}$ in alkaline solutions. It is also seen in Fig. $7 \mathrm{~b}$ that the absolute value of the zeta potential is higher for Pt/Ketjen than for Pt/Vulcan (at same $\mathrm{pH}$ ) when $\mathrm{pH}$ is higher than 5.

The results of the light scattering measurements strongly suggest that the $\mathrm{pH}$ of the catalyst ink controls the surface charge of the catalyst $(\mathrm{Pt} / \mathrm{C})$ particles and thus the stability of the catalyst ink; the electrostatic repulsion between the catalyst particles increases with increasing the catalyst ink $\mathrm{pH}$, stabilizing its dispersion. When the dispersion of the catalyst ink is stable, agglomeration of the catalyst particles is inhibited and uniform catalyst films are obtained. 

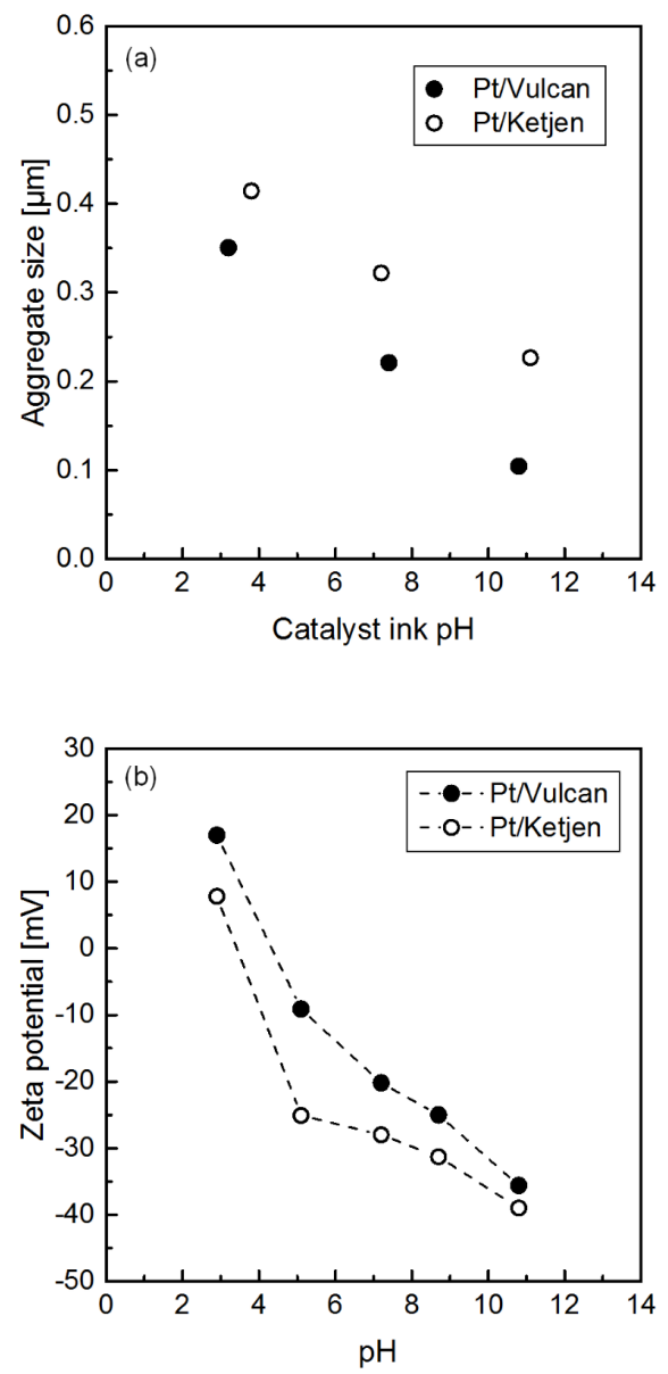

Fig. 7. (a) Aggregate size in the catalyst inks of the $50 \mathrm{wt}$ \% Pt/Vulcan catalyst and the $50 \mathrm{wt}$ \% $\mathrm{Pt} /$ Ketjen catalyst measured by dynamic light scattering. (b) Zeta potential of the catalyst inks of the 50 wt. \% Pt/Vulcan catalyst and the 50 wt. \% Pt/Ketjen catalyst measured by electrophoretic light scattering.

\section{Conclusion}

The influence of the ink $\mathrm{pH}$ of $\mathrm{Pt} / \mathrm{C}$ catalysts on the ORR activity determined by TF-RDE technique was studied. The results of the light scattering measurements suggest aggregate size of the Pt/C catalyst particles in the catalyst inks decreases with increasing $\mathrm{pH}$ of the ink due to 
an increase in the magnitude of the zeta potential. It seems that the agglomeration of the catalyst particles does not affect the utilization of the Pt NPs (i.e. accessibility of the electrolyte solution to the Pt NPs), because the ECSA values are unaffected by catalyst ink $\mathrm{pH}$. Meanwhile, the measured ORR specific activity is highly affected by agglomeration of the catalyst particles. We assume that the reduced ORR activity in the case of agglomerated dispersions is due to mass transport resistance, such as $\mathrm{O}_{2}$ diffusion resistance and protonic resistance, within the catalyst layer. This phenomenon is well recognized in the preparation of MEAs for PEMFCs [27]. However, the influence of the catalyst ink $\mathrm{pH}$ on this effect is widely not discussed in the publicly available literature. Only patent literature reports an influence of catalyst ink $\mathrm{pH}$ on its dispersion and the performance of MEAs at a high current density region $(0.6-0.4 \mathrm{~V})$ [19]. To our knowledge, the influence of the catalyst ink $\mathrm{pH}$ is not discussed in catalyst screening using the TF-RDE method. Our results demonstrate that the impact of the agglomeration of the catalyst particles on the mass transfer resistance can be significant even at high potentials ( 0.9 V), where the ORR activity of the catalysts is usually measured by the TF-RDE method. This is an important finding and suggests that TF-RDE measurements indeed mimic MEA behavior. On the other hand, it means that simple screening tests using the TF-RDE technique can be misleading if the ink is not optimized for the individual catalyst material. Especially the $\mathrm{pH}$ of the catalyst ink is an important parameter that needs to be carefully controlled and optimized in order to measure the intrinsic ORR activity of high surface area catalysts by the TF-RDE technique.

\section{Acknowledgments}

We acknowledge the collaboration with Prof. L. T. Kuhn, Dr. S. B. Simonsen, and Prof. J. K. Kirkensgaard concerning the TEM and SAXS analysis of our nanoparticle samples. Anton Paar 
is acknowledged for preliminary DLS measurements of our samples and Dipl.-Ing. Andrea Mingers of the group of Prof. Karl J.J. Mayrhofer at the MPIE for the ICP-MS analysis. J.Q. and M.A. acknowledge support from the Villum Foundation in form of a block stipend.

\section{References}

[1] T.J. Schmidt, H.A. Gasteiger, G.D. Stäb, P.M. Urban, D.M. Kolb, R.J. Behm, Characterization of High-Surface-Area Electrocatalysts Using a Rotating Disk Electrode Configuration, J. Electrochem. Soc. 145 (1998) 2354.

[2] H.A. Gasteiger, S.S. Kocha, B. Sompalli, F.T. Wagner, Activity benchmarks and requirements for Pt, Pt-alloy, and non-Pt oxygen reduction catalysts for PEMFCs, Appl. Catal. B Environ. 56 (2005) 9. doi:10.1016/j.apcatb.2004.06.021.

[3] K.J.J. Mayrhofer, D. Strmcnik, B.B. Blizanac, V. Stamenkovic, M. Arenz, N.M. Markovic, Measurement of oxygen reduction activities via the rotating disc electrode method: From Pt model surfaces to carbon-supported high surface area catalysts, Electrochim. Acta. 53 (2008) 3181. doi:10.1016/j.electacta.2007.11.057.

[4] K. Hartl, K.J.J. Mayrhofer, M. Lopez, D. Goia, M. Arenz, AuPt core-shell nanocatalysts with bulk Pt activity, Electrochem. Commun. $12 \quad$ (2010) 1487. doi:10.1016/j.elecom.2010.08.013.

[5] K. Shinozaki, J.W. Zack, S. Pylypenko, B.S. Pivovar, S.S. Kocha, Oxygen Reduction Reaction Measurements on Platinum Electrocatalysts Utilizing Rotating Disk Electrode Technique, J. Electrochem. Soc. 162 (2015) F1384. doi:10.1149/2.0551512jes.

[6] A.J. Bard, L.R. Faulkner, Electrochemical Methods: Fundamentals and Applications, 2nd edi., John Wiley \& Sons, New York, 2001. 
[7] E. Higuchi, H. Uchida, M. Watanabe, Effect of loading level in platinum-dispersed carbon black electrocatalysts on oxygen reduction activity evaluated by rotating disk electrode, J. Electroanal. Chem. 583 (2005) 69. doi:10.1016/j.jelechem.2005.01.041.

[8] I. Takahashi, S.S. Kocha, Examination of the activity and durability of PEMFC catalysts in liquid electrolytes, J. Power Sources. $195 \quad$ (2010) 6312. doi:10.1016/j.jpowsour.2010.04.052.

[9] K. Shinozaki, J.W. Zack, R.M. Richards, B.S. Pivovar, S.S. Kocha, Oxygen Reduction Reaction Measurements on Platinum Electrocatalysts Utilizing Rotating Disk Electrode Technique, J. Electrochem. Soc. 162 (2015) F1144. doi:10.1149/2.1071509jes.

[10] Y. Garsany, J. Ge, J. St-Pierre, R. Rocheleau, K.E. Swider-Lyons, Analytical Procedure for Accurate Comparison of Rotating Disk Electrode Results for the Oxygen Reduction Activity of Pt/C, J. Electrochem. Soc. 161 (2014) F628. doi:10.1149/2.036405jes.

[11] Y. Garsany, I.L. Singer, K.E. Swider-Lyons, Impact of film drying procedures on RDE characterization of Pt/VC electrocatalysts, J. Electroanal. Chem. 662 (2011) 396. doi:10.1016/j.jelechem.2011.09.016.

[12] K. Ke, K. Hiroshima, Y. Kamitaka, T. Hatanaka, Y. Morimoto, An accurate evaluation for the activity of nano-sized electrocatalysts by a thin-film rotating disk electrode: Oxygen reduction on $\mathrm{Pt} / \mathrm{C}$, Electrochim. Acta. $72 \quad$ (2012) 120. doi:10.1016/j.electacta.2012.04.004.

[13] S.S. Kocha, J.W. Zack, S.M. Alia, K.C. Neyerlin, B.S. Pivovar, Influence of Ink Composition on the Electrochemical Properties of Pt/C Electrocatalysts, ECS Trans. 50 (2012) 1475.

[14] J. Kim, S.W. Lee, C. Carlton, Y. Shao-Horn, Oxygen Reduction Activity of PtxNi1-x Alloy Nanoparticles on Multiwall Carbon Nanotubes, Electrochem. Solid-State Lett. 14 
(2011) B110. doi:10.1149/1.3613677.

[15] E. Higuchi, A. Taguchi, K. Hayashi, H. Inoue, Electrocatalytic activity for oxygen reduction reaction of Pt nanoparticle catalysts with narrow size distribution prepared from $(\mathrm{n}=3-8) \quad$ complexes, J. Electroanal. Chem. $663 \quad$ (2011) 84. doi:10.1016/j.jelechem.2011.09.028.

[16] O.J. Curnick, B.G. Pollet, P.M. Mendes, Nafion-stabilised Pt/C electrocatalysts with efficient catalyst layer ionomer distribution for proton exchange membrane fuel cells, RSC Adv. 2 (2012) 8368. doi:10.1039/c2ra21071a.

[17] M. Nesselberger, S. Ashton, J.C. Meier, I. Katsounaros, K.J.J. Mayrhofer, M. Arenz, The Particle Size Effect on the Oxygen Reduction Reaction Activity of Pt Catalysts: Influence of Electrolyte and Relation to Single Crystal Models, J. Am. Chem. Soc. 133 (2011) 17428. doi:Doi 10.1021/Ja207016u.

[18] J. Speder, L. Altmann, M. Roefzaad, M. Bäumer, J.J.K. Kirkensgaard, K. Mortensen, M. Arenz, Pt based PEMFC catalysts prepared from colloidal particle suspensions--a toolbox for model studies., Phys. Chem. Chem. Phys. 15 (2013) 3602. doi:10.1039/c3cp50195g.

[19] J.E. O’Hara, Altering zeta potential of dispersions for better hcd performance and dispersion stability, US7399549B2, 2008.

[20] Y. Wang, J. Ren, K. Deng, L. Gui, Y. Tang, Preparation of Tractable Platinum , Rhodium , and Ruthenium Nanoclusters with Small Particle Size in Organic Media Preparation of Tractable Platinum, Rhodium , and Ruthenium Nanoclusters with Small Particle Size in Organic Media, Chem. Mater. 12 (2000) 1622. doi:10.1021/cm0000853.

[21] J. Speder, A. Zana, I. Spanos, J.J.K. Kirkensgaard, K. Mortensen, M. Hanzlik, M. Arenz, Comparative degradation study of carbon supported proton exchange membrane fuel cell 
electrocatalysts - The influence of the platinum to carbon ratio on the degradation rate, J. Power Sources. 261 (2014) 14. doi:10.1016/j.jpowsour.2014.03.039.

[22] J. Speder, L. Altmann, M. Bäumer, J.J.K. Kirkensgaard, K. Mortensen, M. Arenz, The particle proximity effect: from model to high surface area fuel cell catalysts, RSC Adv. 4 (2014) 14971. doi:10.1039/c4ra00261j.

[23] K.J.J. Mayrhofer, G.K.H. Wiberg, M. Arenz, Impact of Glass Corrosion on the Electrocatalysis on Pt Electrodes in Alkaline Electrolyte, J. Electrochem. Soc. 155 (2008) P1. doi:10.1149/1.2800752.

[24] I. Schrader, J. Warneke, S. Neumann, S. Grotheer, A.A. Swane, J.J.K. Kirkensgaard, M. Arenz, S. Kunz, Surface Chemistry of "Unprotected" Nanoparticles: A Spectroscopic Investigation on Colloidal Particles, J. Phys. Chem. C. 119 (2015) 17655. doi:10.1021/acs.jpcc.5b03863.

[25] H. Jinnai, R.J. Spontak, T. Nishi, Transmission electron microtomography and polymer nanostructures, Macromolecules. 43 (2010) 1675. doi:10.1021/ma902035p.

[26] Q. Lü, A. Li, F. Guo, L. Sun, L. Zhao, Experimental study on the surface modification of $\mathrm{Y}(2) \mathrm{O}(3): \mathrm{Tm}\left(3^{+}\right) / \mathrm{Yb}\left(3^{+}\right)$nanoparticles to enhance upconversion fluorescence and $\begin{array}{lllll}\text { weaken } & \text { aggregation., } & \text { Nanotechnology. } & 19 & \text { (2008) }\end{array}$ doi:10.1088/0957-4484/19/14/145701.

[27] M. Uchida, Y. Aoyama, N. Eda, A. Ohta, Investigation of the Microstructure in the Catalyst Layer and Effects of Both Perfluorosulfonate Ionomer and PTFE-Loaded Carbon on the Catalyst Layer of Polymer Electrolyte Fuel Cells, J. Electrochem. Soc. $142(1995) 4143$. 


\section{Figure captions}

Figure 1. Comparison of our previous data and the presented work where NF-SIPAD technique was employed for the catalyst film fabrication. In both work, the commercial $46 \mathrm{wt}$ \% $\mathrm{Pt} / \mathrm{C}$ catalyst (TKK) was measured by RDE employing identical condition and experimental procedure. Our previous data is taken from reference [17].

Figure 2. (a) Scheme of the Pt/C catalyst synthesis via tool-box synthesis approach and TEM images of (b) 50 wt. \% Pt/Vulcan catalys and (c) 50 wt. \% Pt/Ketjen catalyst that are obtained from the synthesis approach.

Figure 3. Photographs of catalyst inks and catalyst films fabricated from each catalyst ink: (a)(b) the commercial 46 wt. \% Pt/C catalyst (TKK), IPA:DI water (1:3, v:v) as ink solvent, ink drying in IPA atmosphere, (c)(d) the homemade 50 wt. \% Pt/Vulcan catalyst, $\mathrm{H}_{2} \mathrm{O}$ as ink solvent, ink drying under $\mathrm{N}_{2}$ flow, (e)(f) the homemade50 wt. \% Pt/Vulcan catalyst, IPA:DI water (1:3, v:v) as ink solvent, ink drying in IPA atmosphere. The catalyst films were fabricated on the GC electrodes and thereafter transferred on a paper before taking the photographs.

Figure 4. Photographs of catalyst inks of the homemade 50 wt. \% Pt/Vulcan catalyst with different $\mathrm{pH}$ and catalyst films fabricated from each catalyst ink: (a)(b) ink $\mathrm{pH}=4.3$, without adding $\mathrm{KOH}$, (c)(d) ink $\mathrm{pH}=8.0, \mathrm{KOH}$ added, (e)(f) ink $\mathrm{pH}=11.3, \mathrm{KOH}$ added. The catalyst films were fabricated on the GC electrodes and thereafter transferred on a paper before taking the photographs. 
Figure 5. Comparison of voltammograms for the catalyst films fabricated from the catalyst inks of the homemade 50 wt. \% Pt/Vulcan catalyst with different $\mathrm{pH}(\mathrm{pH}=4.3,8.0,11.3)$ : (a) cyclic voltammograms in Ar-purged $0.1 \mathrm{M} \mathrm{HClO}_{4}$ at the scan rate of $50 \mathrm{mV} \mathrm{s}^{-1}$. The neutral ink $(\mathrm{pH}=$ 8.0) is not shown for the clarity. (b) linear sweep voltammograms (positive scans) in $\mathrm{O}_{2}$-purged $0.1 \mathrm{M} \mathrm{HClO}_{4}$ at the scan rate of $50 \mathrm{mV} \mathrm{s}^{-1}$ and the rotation speed of $1600 \mathrm{rpm}$. $R_{\text {sol }}$ and background were corrected. Inset is Tafel plot obtained from the linear sweep voltammograms.

Figure 6. Influence of $\mathrm{pH}$ of catalyst inks on SA at $0.9 \mathrm{~V}$ vs. RHE and ECSA: (a)(b) the homemade 50 wt. \% Pt/Vulcan catalyst, (c)(d) the homemade 50 wt. \% Pt/Ketjen catalyst, (e)(f) the commercial 46 wt. \% Pt/C catalyst (TKK). At least three measurements were conducted for each catalyst ink.

Figure 7. (a) Aggregate size in the catalyst inks of the $50 \mathrm{wt}$ \% Pt/Vulcan catalyst and the 50 wt. \% Pt/Ketjen catalyst measured by dynamic light scattering. (b) Zeta potential of the catalyst inks of the $50 \mathrm{wt}$ \% Pt/Vulcan catalyst and the $50 \mathrm{wt}$ \% Pt/Ketjen catalyst measured by electrophoretic light scattering. 